\title{
Nota de Terapéutica: Utilizando el puntaje de Framingham para la evaluación del riesgo cardiovascular en atención primaria
}

Using Framingham for primary prevention cardiovascular risk assessment. Letter, issue 63, March - April 2007

Traducido con autorización de "Therapeutics Initiative" (The University of British Columbia). Disponible en URL: http://www.ti.ubc.ca/PDF/63.pdf

Utilizando los resultados de un meta-análisis, la anterior nota farmacológica de Evidencia (traducción de la carta terapéutica número 62 ) delineó las bases para estimar los beneficios potenciales de tratar la hipertensión leve

Otra aproximación a la estimación del riesgo cardiovascular utilizada por muchos clínicos es la calculadora de riesgo cardiovascular (RCV) de Framingham, que incluye la edad, el sexo, el nivel de colesterol total, el nivel de colesterol HDL, la presión arterial sistólica, la presencia o ausencia de diabetes mellitus, y el status postivo o negativo de fumador, a través de una fórmula matemática para intentar predecir el RCV. En esta fórmula no se encuentran incluídos factores de riesgo más novedosos -proteína $C$ reactiva (PCR) LpPLA, IL-6, Dímero D, homocisteinemia, niveles sanguíneos de folatos, vitamina B6, leptina o plasminógeno- $y$, de hecho, una publicación reciente concluye que su inclusión rutinaria no es necesaria para la evaluación del RCV. Otro estudio sugiere que el uso del cociente APOB/A-I no agrega mucho valor al puntaje de Framingham en relación a la predicción del RCV . Como en muchas calculadoras clínicas, no se incluyen como estimadores de riesgo la historia familiar de enfermedad coronaria ni el peso corporal, por lo que el médico debería usar su juicio clínico en los casos en que considere que estos factores son importantes para predecir el RCV.

¿Qué estamos calculando con una calculadora de Framingham?

Algunas calculadoras de Framingham calculan el RCV global (RCVG) mientras que otras calculan el riesgo de enfermedad coronaria (REC). En este caso, el RCVG fue definido como angina de pecho estable o inestable, infarto agudo de miocardio (IAM) o muerte por enfermedad coronaria, habiendo utilizado estas definiciones las primeras tablas para cálculo del riesgo. Las calculadoras de riesgo más recientes calculan el riesgo de puntos finales "duros" de enfermedad coronaria (IAM y muerte por enfermedad cardiovascular) que corresponden a entre los dos tercios y los tres cuartos del total de los casos de enfermedad coronaria.

Para las reglas de Framingham, un evento cardiovascular se define como IAM, angina de reciente comienzo, accidente cerebro vascular isquémico transitorio (AIT) o definitivo (ACV) enfermedad vascular periférica (EVP) insuficiencia cardíaca congestiva (ICC) y muerte relacionada a una causa cardiovascular.

Para resumir, podemos decir que si usamos una calculadora, deberíamos asegurarnos de comprender qué estamos calculando.

\section{¿En qué período de tiempo se está calculando el riesgo?}

La mayoría de las calculadoras calculan el riesgo a cinco años o diez años, (aproximadamente el doble en diez que en cinco). A pesar de que algunos defienden la conveniencia de calcular el RCV a tiempos más prolongados (como el de toda la vida del individuo) esta estrategia no es de mucha ayuda ya que la mayoría de la gente tendrá un "alto" riesgo de enfermedad coronaria si se lo calcula hasta el final de su vida.

Otra aproximación, ha sido la utilización de estas calculadoras para mostrarles a los pacientes cómo se compara su RCV en relación a otros pacientes de una edad similar, pero sin el resto de los factores de riesgo. Por, último, algunos investigadores han desarrollado modelos de riesgo que pueden estimar el cambio en "la edad cardiovascular" que se produce al modificar determinados factores de riesgo específicos
¿Cuál sería la estimación del riesgo de enfermedad coronaria y del riesgo cardiovascular en un paciente modelo si usáramos la fórmula de Framingham?

El señor EBP es un hombre de 55 años con una presión arterial de $146 / 94 \mathrm{mmHg}$, no tiene diabetes ni fuma.

A pesar de que al señor EBP no se le solicitaron mediciones sanguíneas de colesterol, asumamos que su colesterol total es de $190 \mathrm{mg} / \mathrm{dL}$ y que su colesterol HDL es de $48,75 \mathrm{mg} / \mathrm{dL}$

De acuerdo a los datos de un meta-análisis de cinco ensayos clínicos que incluyeron pacientes con una presión arterial sistólica promedio de 160/98 mmHg (carta terapéutica número 62) comparándola con el uso de placebo, la terapia farmacológica redujo entre 3,1 y $4 \%$ el riesgo a cinco años de un punto final combinado de ACV totales más IAM.

Utilizando la calculadora de Framingham habriamos predicho para nuestro paciente un RCVG a diez años de $16 \%$ y de enfermedad coronaria de $11 \%$ (ver tabla 1 ).

En nuestro artículo anterior, la incidencia resultante del metaanálisis de los cinco estudios -habían incluido 54\% de varones con una edad promedio de 51 años, una presión arterial promedio $160 / 98 \mathrm{mmHg}$, la mayoría sin evidencia de enfermedad cardiovascular- la incidencia combinada de IAM y ACV fue de $4 \%$ a cinco años o de $8 \%$ a diez años. La estimación de a través del puntaje de Framingham resulta mayor (16\%) pero incluye más puntos finales: angina de reciente comienzo, AIT, EVP e ICC.

En las siguientes tablas se presentan algunos de los números resultantes del cálculo con el puntaje de Framingham, utilizando fórmulas publicadas en hojas de cálculo. Las columnas en negrita son las que corresponden a nuestro paciente, presentándose el resto para mostrar el impacto sobre el riesgo estimado, de los cambios en la presión arterial sistólica o en el colesterol total.

Tabla 1: efecto de la presión arterial en el riesgo cardiovascular a diez años de un varón de 55 años, no fumador, no diabético y con un colesterol total de $190 \mathrm{mg} / \mathrm{dL}$ y $\mathrm{HDL}$ de $48,75 \mathrm{mg} / \mathrm{dL}$.

\begin{tabular}{l|c|c|c|}
\hline Tensión arterial sistólica & $136 \mathrm{mmHg}$ & $\mathbf{1 4 6} \mathbf{~ m m H g}$ & $156 \mathrm{mmHg}$ \\
\hline Riesgo cardiovascular global & $14 \%$ & $\mathbf{1 6} \%$ & $18 \%$ \\
\hline Riesgo de enfermedad coronaria & $\mathbf{9} \%$ & $\mathbf{1 1 \%}$ & $12 \%$ \\
\hline
\end{tabular}

Tabla 2: efecto del colesterol en el riesgo a diez años de un varón de 55 años no fumador, no diabético, con una presión arterial sistólica de $146 \mathrm{mmHg}$ y un colesterol $\mathrm{HDL}$ de $48,75 \mathrm{mg} / \mathrm{dL}$.

\begin{tabular}{l|c|c|c}
\hline Colesterol total & $152 \mathbf{~ m g} / \mathrm{dL}$ & $\mathbf{1 9 0} \mathbf{~ m g} / \mathbf{d L}$ & $228 \mathrm{mg} / \mathrm{dL}$ \\
\hline Riesgo cardiovascular global & $13 \%$ & $\mathbf{1 6} \%$ & $19 \%$ \\
\hline Riesgo de enfermedad coronaria & $8 \%$ & $\mathbf{1 1} \%$ & $13 \%$ \\
\hline
\end{tabular}

¿Cómo podemos usar estos números para estimar el beneficio del tratamiento?

\section{Esto requiere extrapolar datos desde la evidencia}

A. Podríamos simplemente utilizar la tabla y estimar qué pasaría si el paciente tuviera una presión arterial de $136 \mathrm{mmHg}$ en vez de 146 mmHg: el RCVG a diez años descendería de 16 a 14\% y el RCVG a cinco años descendería de 8 a $7 \%$, resultando una reducción de riesgo absoluto (RRA) del $1 \%$.

B. Podríamos tomar el RCVG predicho a diez años del $16 \%$ y 
convertirlo en un riesgo a cinco años de $8 \%$. Luego lo multiplicaríamos por la reducción de riesgo relativo promedio (RRR) del $31 \%$ (IC95\% 25 a 37) a cinco años para el punto final combinado IAM-ACV, estimación extraída de un meta-análisis de todos los ensayos clínicos de drogas antihipertensivas. Esto predice una disminución del 2,4\% del riesgo absoluto de enfermedad coronaria.

Para el tratamiento con estatinas, los ensayos clínicos de prevención primaria han demostrado una disminución del 25\% (IC95\% 19 a 32) del REC en hombres pero no en mujeres -ver carta terapéutica número 48. Para este hombre, su REC a cinco años es de $5,5 \%$ (la mitad del riesgo de REC a diez años) y por lo tanto se puede predecir un beneficio absoluto del tratamiento con estatinas de $1,4 \%$.

Muchos grupos han definido porcentajes específicos de riesgo o umbrales de tratamiento de presión arterial y colesterol. Sin embargo, estos umbrales son todos arbitrarios y basados solamente en opiniones, ya que raramente toman en cuenta las preferencias de los pacientes individuales.

En este sentido, un estudio sugirió que menos de un tercio de los pacientes, con o sin antecedente de enfermedad coronaria, tomaría una droga "segura", si la reducción del riesgo absoluto de IAM a cinco años fuera menor o igual a $5 \%$. Por otro lado, un estudio que reportó las preferencias de profesionales de la salud y de la población general concluyó que las decisiones personales frecuentemente colisionan con las recomendaciones de las guías "basadas en la evidencia"

¿Ha hecho alguien una revisión sistemática sobre la precisión y el impacto clínico de la evaluación del riesgo cardiovascular?

Sí fue hecha y fueron encontrados 17 estudios epidemiológicos que habían evaluado la precisión de los puntajes de Framingham, concluyendo dicha revisión sistemática que estos no eran precisos y que tendían a subestimar el riesgo en poblaciones de alto riesgo y a sobrestimarlo en las de bajo riesgo. También fueron encontrados cuatro ensayos clínicos que habian valorado el impacto de la evaluación del RCV mostrando un bajo impacto en el número total de personas tratadas.

De todas maneras, si realmente queremos comprometernos en la toma de decisiones compartida con el paciente, éste necesitará datos que le permitan hacerse una idea del riesgo que corre y de los potenciales beneficios de un tratamiento. O sea que, a pesar de sus limitaciones, el uso de los puntajes de Framingham o de algún otro medio para calcular del RCV, permite a los médicos alguna aproximación objetiva al problema, proveyendo por lo menos mayor información que el simple uso de un nivel aislado de presión arterial o de colesterol para identificar a los pacientes que requieren tratamiento.

Sin embargo, los profesionales de la salud debemos estar al tanto de que no se ha realizado una extensa validación de estas herramientas, por lo menos en la población canadiense.

¿Cuáles son algunas de las limitaciones de las calculadoras de riesgo de Framingham?

- No predicen el riesgo más allá de 12 años.

- No proveen una estimación de la precisión de su cálculo (intervalos de confianza).

- Son menos precisas para la estimación en pacientes con valores extremos en sus factores de riesgo (muy altos o muy bajos) - Si nuestro paciente es diferente de la población estudiada, la evaluación del riesgo es más imprecisa. Por ejemplo, en población no norteamericana, hombres japoneses o hispanos, mujeres aborígenes, personas menores de 30 años o mayores de 65 y diabéticos

\section{Conclusiones}

Si usamos el puntaje de Framingham para calcular el riesgo, tenemos que asegurarnos de conocer los puntos finales incluidos y el período de seguimiento.

La evaluación del RCV tiene una serie de limitaciones, faltando evidencia sobre su impacto en las prácticas prescriptivas o en puntos finales de salud.

La utilidad de la estrategia de "evaluación del RCV" necesita ser evaluada mediante ensayos clínicos aleatorizados, en los que se la compare con el manejo usual, con el objetivo de determinar el impacto sobre la práctica prescriptiva en poblaciones con diferentes riesgos basales y para documentar si los pacientes a quienes se les presentan estimaciones de su RCV se sienten más informados y toman decisiones diferentes sobre su tratamiento.

El borrador de esta carta terpéutica fue enviada para su revisión a 40 expertos y médicos de atención primaria para corregir cualquier inexactitud y para asegurar que la información fuera concisa y relevante para los médicos.

\section{Referencias}

1. Folsom AR, Chambless LE, Ballantyne CM, et al. An assessment of incremental coronary risk prediction using C-reactive protein and other novel risk markers: the atherosclerosis risk in communities study. Arch Intern Med. 2006; 166(13):1368-73.

2. van der Steeg WA, Boekholdt SM, Stein EA, et al. Role of the apolipoprotein B-apolipoprotein A-I ratio in cardiovascular risk assessment: a case-control analysis in the EPICNorfolk. Ann

Intern Med. 2007; 146:640-8

3. Grover SA, Paquet S, Levinton C, et al. Estimating the benefits of modifying risk factors of cardiovascular disease: A comparison of primary vs. secondary prevention. Arch Intern Med. 1998;158:655-662.

4. Mulrow C, Lau J, Cornell J, Brand M. Pharmacotherapy for hypertension in the elderly. Cochrane Database of Systematic Reviews. 1998; Issue 2. Art. No.: CD000028. DOI: $10.1002 / 14651858$

5. Trewby PN, Reddy AV, Trewby CS, et al. Are preventive drugs preventive-enough? A study of patients' expectation of benefit from preventive drugs. Clin Med. 2002; 2(6):527-33.

6. Lewis DK, Robinson J, Wilkinson E. Factors involved in deciding to start preventive treatment: qualitative study of clinicians' and lay people's attitudes. BMJ. 2003; $327(7419): 841-5$

7. Brindle P, Beswick A, Fahey T, Ebrahim S. Accuracy and impact of risk assessment in the primary prevention of cardiovascular disease: a systematic review. Heart. 2006; 92(12):1752-9

8. Grover SA, Hemmelgarn B, Joseph L, Milot A, Tremblay G. The role of global risk assessment in hypertension therapy. Can J Cardiol 2006. 22(7):606-13. 